\title{
The cryogenic control system of SHINE
}

\author{
Geyang Jiang ${ }^{1} \mathbb{D}$, Qing $\mathrm{Ni}^{2}$, Shuhua Wang ${ }^{1}$, Jiuce Sun ${ }^{2}$, Zhengrong Ouyang ${ }^{1 *}$, Lei Zhang ${ }^{2}$, Zilei Tong ${ }^{1}$ and Yi Wang ${ }^{1}$
}

\author{
* Correspondence: oyo@ipp.ac.cn \\ 'Shanghai Advanced Research \\ Institute, Chinese Academy of \\ Sciences, No. 99 Haike Road, \\ Zhangjiang Hi-Tech Park, Pudong, \\ Shanghai, China \\ Full list of author information is \\ available at the end of the article
}

\begin{abstract}
The Shanghai High repetition rate XFEL and Extreme Light Facility (SHINE), an advanced XFEL project, is now being built at Shanghai Advanced Research Institute (SARI), Chinese Academy of Sciences. It includes a hard X-ray free electron laser and a $100 \mathrm{pW}$ intense laser facilities with overall length of $3.1 \mathrm{~km}$. The XFEL part including an $8 \mathrm{GeV}$ LINAC and 3 undulator lines is cooled with forced superfluid and supercritical helium at $2 \mathrm{~K} / 4 \mathrm{~K}$. The cryogenic system of SHINE consists of test facility cryogenic system (TFCS), accelerator cryogenic system (ACCS), and undulator cryogenic system (UNCS). A dedicated control system based on Experimental Physics and Industrial Control System (EPICS) will be built to automate the cryogenic system with process control, PID control loops, real-time data acquisition and storage, alarm handler and human machine interface. It is capable of automatic recovery as well. This paper describes details of control system structure, interfaces, controllers and the integration under EPICS framework.
\end{abstract}

Keywords: Cryogenic control, PLC, EPICS, Phoebus, Interlock

\section{Introduction}

The SHINE cryogenic system is built to provide sufficient cooling at appropriate temperature levels to enable the test and operation of the superconducting components of accelerator and undulator system respectively. It is divided into three parts, TFCS, ACCS and UNCS. Each part consists of three major subsystems: cryogenic plant, distribution system and associated auxiliary systems (including Helium gas recovery, storage and purification). The TFCS will be built to test the superconducting cavity, tuner, BPM, input coupler, $2 \mathrm{~K}$ heat exchanger, and assembled cryomodule, etc. According to the project construction sequence, the TFCS will be the first of the SHINE cryogenic system to be designed, installed and commissioned.

The ACCS will generate and transfer all the required cooling to the LINAC containing seventy five $1.3 \mathrm{GHz}$ and two $3.9 \mathrm{GHz}$ cryomodules. To archive the overall $2 \mathrm{~K}$ cooling capacity of $12 \mathrm{~kW}$, the ACCS adopts three individual cryoplants with state-ofthe-art technology. Each cryoplant could provide an approximate $4 \mathrm{~kW}$ cooling power at $2 \mathrm{~K}$ and an overall equivalent cooling power of $18 \mathrm{~kW}$ at $4.5 \mathrm{~K}$. Accordingly, the LINAC will also be divided into three strings: the upstream string, the midstream string, and the downstream string. The accelerator cryoplants (ACCP) will be located at the two ends of the LINAC (around $2 \mathrm{~km}$ distance). Among three cryoplants, two

(c) The Author(s); licensee Springer on behalf of EPJ. 2021 Open Access This article is licensed under a Creative Commons Attribution 4.0 International License, which permits use, sharing, adaptation, distribution and reproduction in any medium or format, as long as you give appropriate credit to the original author(s) and the source, provide a link to the Creative Commons licence, and indicate if changes were made. The images or other third party material in this article are included in the article's Creative Commons licence, unless indicated otherwise in a credit line to the material. If material is not included in the article's Creative Commons licence and your intended use is not permitted by statutory regulation or exceeds the permitted use, you will need to obtain permission directly from the copyright holder. To view a copy of this licence, visit http://creativecommons.org/licenses/by/4.0/. 
cryoplants will be installed at the beginning of the LINAC to feed the upstream and midstream string by independent cryogenic distribution systems, and the third one will be located at the end of the LINAC to feed the downstream cryomodule string [1]. The layout of SHINE ACCS and LINAC are shown in Fig. 1 as follows:

The UNCS will provide the cooling to the 40 Superconducting undulators. The cooling requirements of undulators are rather different from the cryomodules. Therefore, a separate conventional $4.5 \mathrm{~K}$ Helium cryoplant with additional sub cooling and circulating system will be designed and constructed to fulfill the cooling requirements of the undulators. At current stage, our design and construction only focus and cover ACCS part.

The UNCS and ACCS will share a common Helium gas recovery, purification, and inventory system. Separate liquid Helium storage Dewar will be equipped for those three plants to serve as a thermal buffer in one aspect and provide liquid Helium to the experiment station in the other aspect. The TFCS contains a separate gas storage and purification system, however, it could be linked to UNCS and ACCS by distribution lines.

The control system is aimed to implement all devices control and integerates them all. For each sub cryogenic system, refrigerator cold box will be purchased from the vendor-ALAT Company, which performs the cryogenic plant control through Siemens PLCs, mainly model is S7-1500, Profinet and WinCC software. The controls of auxiliary systems supplied by domestic manufacturers is assigned by using Siemens PLCs too. Other controls, such as distribution system, vacuum, etc. are designed and implemented by SARI using Siemens S7-1500/et200SP model PLCs. Up till now, TFCS is being installed and on commissioning at the end of 2020, so next chapters will focus on more details on TFCS control system. Since the whole cryogenic controls is

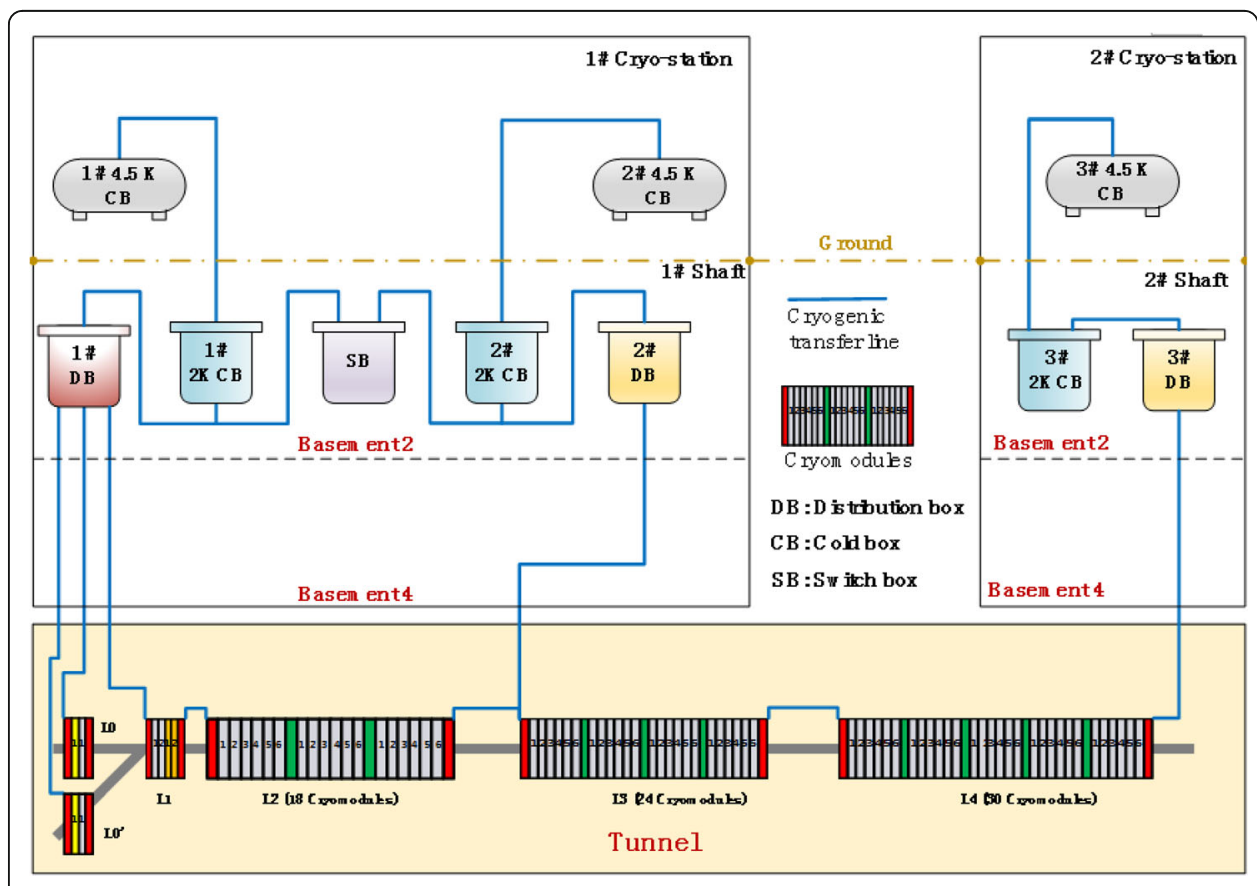

Fig. 1 Simplified layout of SHINE ACCS 
designed scalable, the whole system will be easy to expand based on technical verification after TFCS station construction.

\section{Control system overview}

The cryogenic control system for SHINE is designed to clarifying subsystems including helium compressors, refrigerators, auxiliary subsystems, distribution subsystem, liquid nitrogen, vacuum subsystem, interlock safety, etc. These sub systems can be installed and tested separately and combined into the top control system to accomplish supervisory control, automation, and operational analysis. All essential parameters for the operation of the cryogenic plant shall be monitored and controlled complying with the SHINE technical and safety specifications. Also, the cryogenic control system should be designed to interface with the SHINE central control system. To achieve stability, flexibility, high performance and minimize long-term costs of maintenance for software and hardware, it will be built by using industrial controllers and based on EPICS.

As shown in Fig. 2, the control system will adopt the standard three layers control architecture to ensure scalability and avoid performance limitations. EPICS will give a set of toolkits with well-defined interfaces at both the servers and clients to enable integration and development. SIEMENS fiber Ethernet using TCP/IP protocol will be used to connect all cryogenic control devices.

At the device level, the various components, for example, sensors, actuators and measurement devices are interfaced to local PLCs with touch panel through different types of signal transition.

At the middle layer, PLC will implement most of control logic and PID cycle. Soft IOCs running EPICS runtime database accomplish signal collection from PLC, and convert them to Process Variables (PV) under the rule of EPICS Channel Access (CA) protocol.

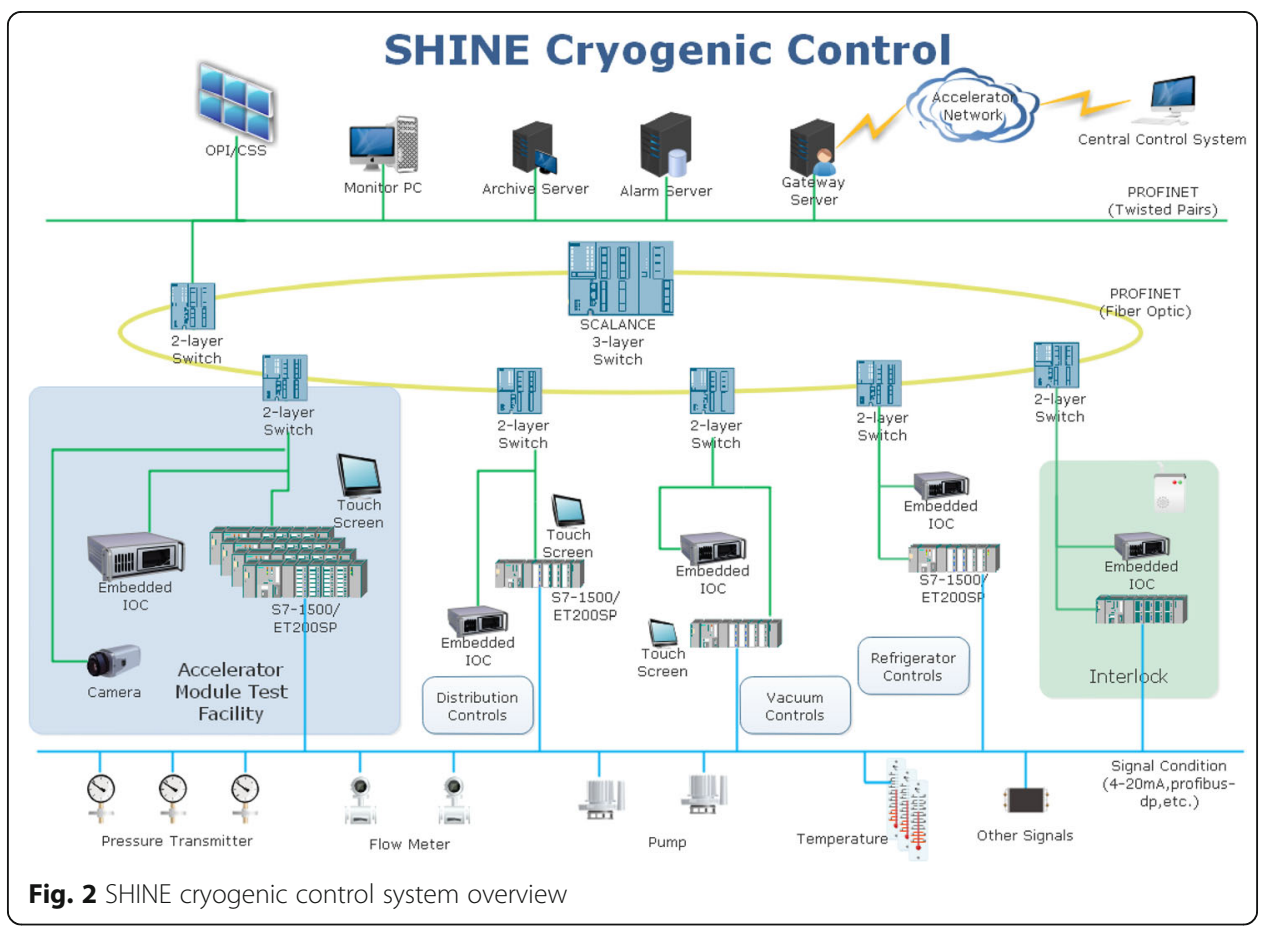


In the core of the control system, powerful Linux servers host the operational files and run other application software based on JAVA or script languages.

With this structure, remote consoles running Graphical User Interfaces (GUI) will allow machine operators to control and optimize the beams and to supervise the state of cryogenic plant in central control room. Since SHINE machine is a long distance LINAC, two key points, Long distance data transfer and scalable need to be considered precisely in system design stage.

\section{Network}

In SHINE cryoplant, ACCS (cryoplant1 and cryoplant2) is distributed in two shafts which is about $2 \mathrm{~km}$ far away. TFCS is about $100 \mathrm{~m}$ nearby cryoplant 1 . In TFCS, there are three halls to accommodate cryogenic devices. As shown in Fig. 3, we choose SIEM ENS Industrial Ethernet switches SCALANCEX with fiber ring to compose backbone network. 3-Layers XM408 switches can support VLAN technology to avoid network storm and full network management functions. Ring network design can reduce network failure possibility [2].

By using 2-Layer switch XC224 and some protocol converters, other field buses such as Profibus, serial bus can be reached from everywhere via Ethernet. The cryogenic control network is designed to operate standalone and integrate into the SHINE central network via EPICS CA gateway to guarantee the performance and not influenced by accelerator network. With this, remote accessing and monitoring are available by exchanging PV data between the accelerator and cryogenic systems.

\section{Interfaces}

Under Industrial Ethernet (IE) structure, all full sub control systems can be connected physically, but interfaces still need to be chosen carefully. We also add a dedicated set of PLC to take the role of all PLC master. There are several kinds of interfaces as Fig. 4:

- interface 1 between PLCs. With same brand SIEMENS PLC, we can use its proprietary protocol to exchange data. There is still a tiny difference between

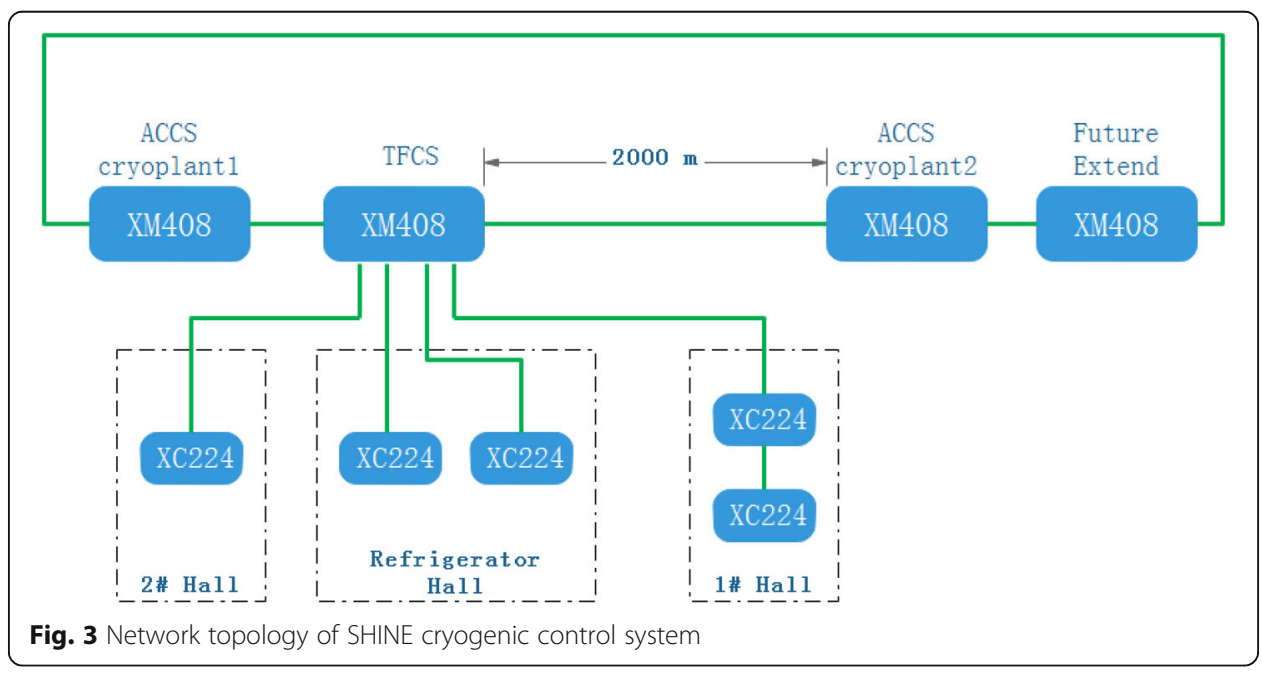




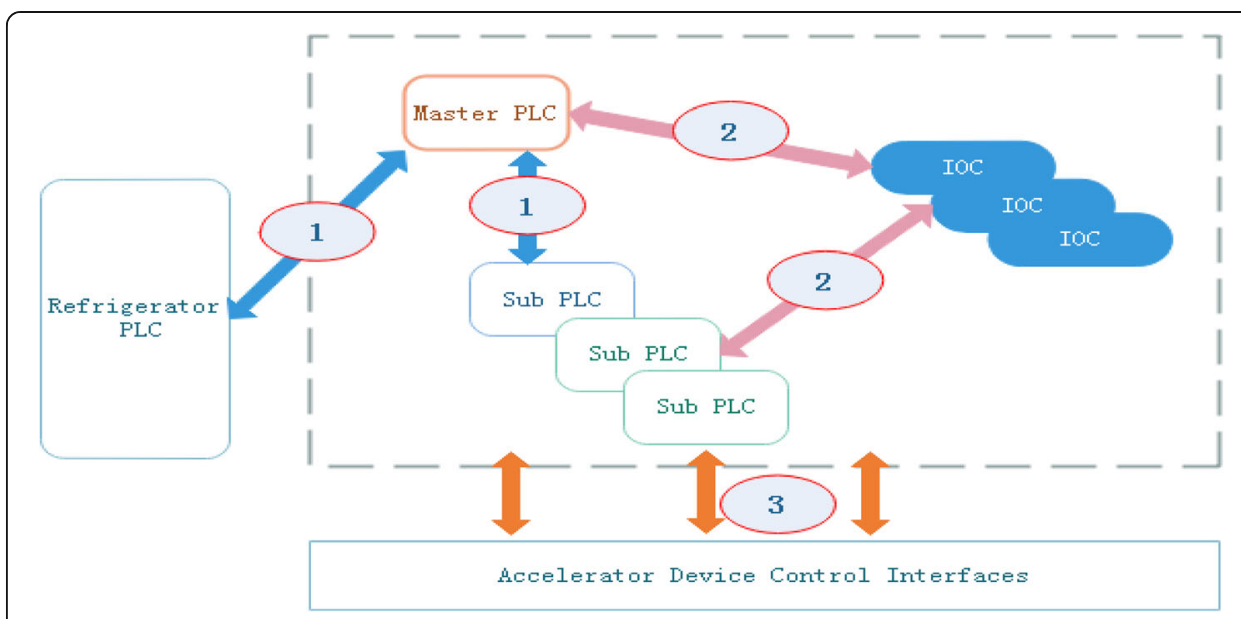

Fig. 4 Interfaces in SHINE cryogenic control system

refrigerator PLC and other sub PLCs. Master to refrigerator is read only with a shared memory table to avoid impact to it; Master to sub is read/write through register address with PLC configuration technology.

- interface 2 between PLCs and IOCs. S7nodave EPICS device support driver resident on IOC is used to implement communication. Unlike other EPICS device supports for the S7 PLCs, this driver program just specifies the memory address in the PLC and the device support uses the ISO-TCP protocol supported by most S7 PLCs in order to exchange data.

- interface 3 between sub PLCs and other accelerator control devices. For example, Modbus TCP protocol is used to communicate to Yokogawa PLC which is used for cryomodule control system. Also electrical signals can be connected to sub PLC via various PLC's modules and cable connections.

\section{Device control}

The SIEMENS Distribution Control System (DCS) offers the software TIA Portal to be used in all PLC stations. These include configuration software, control software, advanced process control software, GUI software for remote computer and local touch panel. Almost all actuators, sensors and transducers' process variables and status information are converted to manipulable and supervisory data by PLCs. Analog/Data conversion, time restricted operation of control algorithm and disposal of input/output variables are realized by PLC as well. To those devices without strict time limit, such as some vacuum signals, circumstance monitor, etc. will be manipulate by IOC. Meanwhile, several kinds of signal condition module with Ethernet port are developed to reduce big mount of long distance cables which originally connecting to PLC rack. At present, TFCS device I/O numbers are shown in Table 1.

There are $1500 \mathrm{I} / \mathrm{O}$ numbers in the control system, under consideration of about $15 \%$ margin. In vacuum/nitrogen system, analog or gauge's value is acquired via network, here is presented as digital/network. If the value exceeds threshold, digital output will trigger warning or interlock. 
Table 1 Control signals amout of SHINE TFCS

\begin{tabular}{lllllll}
\hline I/O & DI & DO & AI & AO & Digital/network & Total \\
\hline Coldbox/refrigerator & 37 & 46 & 150 & 67 & 13 & 313 \\
Compressor/helium recover & 132 & 77 & 105 & 18 & $/$ & 332 \\
Distribution system & 62 & 28 & 278 & 162 & 11 & 541 \\
Vacuum/nitrogen & 15 & 21 & $/$ & $/$ & 27 & 63 \\
Other system & $/$ & $/$ & $/$ & $/$ & 36 & 36 \\
Total & 246 & 172 & 533 & 247 & 87 & 1285 \\
\hline
\end{tabular}

\section{Interlock}

The TFCS interlock system is a bit complicated comparing with future cryoplant interlock system. The refrigerator interlock system is coming with vendor ALAT. The distribution system includes four vertical test cryostats (VTC) to test the Super Conductor (SC) cavities; four horizontal test benches (HTB) to test the assembled cryogenic modules (CM) and magnets; One multi-functional test cryostat (MTC) to test the BPM, coupler, etc.; one undulator test cryostat (UTC) to test the assembled undulator; one backup test cryostat (BTC) to test other potential components such as heat exchanger, etc. All test devices havetheir own interlock signal/logic requirements and these cryostats need to be worked in parallel as possible. The interlock system design strategy is to find the balance between refrigerator shutdown and device safety. Therefore each cryostat control station adopts a separate LADDER program to implement different interlock logic combining with devices locally first. Each cryostat control station has three pairs of DI/DO signal connected to master interlock PLC. Master interlock PLC will execute more complicated safe logic and exchange interlock signals with refrigerator interlock system by electric wire. To ensure strict time limitation and easy connection, a dedicated Complex Programmable Logic Device (CPLD) module with one fiber port, sixteen electric ports and LATTICE chip is developed. With one pair of this module, electric and optical signal can be converted bidirectional in microsecond level [3]. The test results show the time from cryostat control PLC output to master interlock PLC's. Response is about several tens millisecond. Certainly, there are more interlock conditions will be tested, modified and verified during commissioning stage later.

\section{Process control}

The SHINE cryogenic system has several operational modes including cool-down, normal operation, warm-up and quench recovery. During the different operational modes, the low level control logic and loops are written in LADDER or SCL resident in PLC. While in TFCS, especially cryostat controls, the control logic and loops often need to be modified during the test stage. Generally the loops running on the PLCs must work continuously, even momentary interruptions can't be tolerated whereas a short interruption can be tolerated in IOC. Thus for those non critical controls, such as cryostats operational modes will be implemented by State Notation Language (SNL). With this, IOC reboot is allowed for maintenance and modification, PLC will keep the value of all inputs and outputs when the IOC and PLC communication is broken for a while. The EPICS PID record will be used in IOC, which not only allows easy adjustment of the loop constants and changing the loop parameters, but also provides the digital enabling interface to realize the software protection of the cryogenic system. 


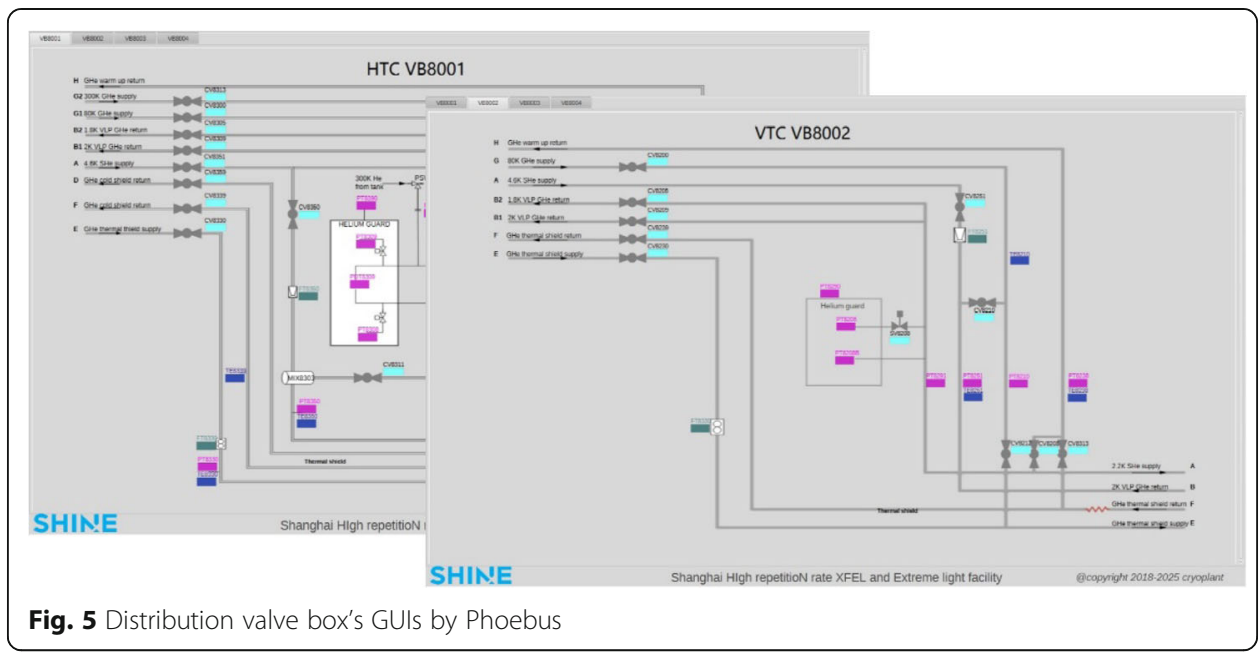

\section{High level software}

As mentioned above, SIEMENS DCS software can fulfil basic system operation and commissioning. EPICS software package is still used to offer more comprehensive functions, such as Archive/Alarm/GUI. Phoebus is an update of the Control System Studio (CSS) toolset. It retains functionality of key CSS and removes dependencies on Eclipse RCP and SWT. This makes it reducing build system complexity and can supporting a fully standalone build process. With its display builder, synaptic displays can be easily made as Fig. 5. It also supports alarm system configuration and related screens (https://control-system-studio. readthedocs.io/en/latest/).

For long-term data storage, we adopt Archiver Appliance, which is an implementation of an archiver for EPICS control systems that aims to archive millions of PVs. One Dell PowerEdge R740 rack server with Redundant Arrays of Independent Disks (RAID) running CENTOS7 and MYSQL database will host all storage (http://slacmshankar. github.io/epicsarchiver_docs/index.html). Operator can navigate history plot by Phoebus or web browser smoothly. The other same configuration Dell server will host other EPICS applications software, for example, NTP server, EPICS base, ELOG system, etc.

\section{Results and discussion}

Up till now, the TFCS control system was designed and under construction. The sub control system has been installed and tested. They are going to be integrated and commissioned in the beginning of 2021. Meanwhile, the conceptual design of ACCS control system has finished. More technician details need to be verified and modified based on TFCS commissionning experience. On this basis, the ACCS control system will be ready for cryoplants first cooling down in the end of 2024.

The authors wish to express their gratitude to the cryogenic group at IHEP for their skillful work and assistance during the design of the cryogenic control system.

\section{Abbreviations}

SHINE: Shanghai High repetition rate XFEL and Extreme Light Facility; SARI: Shanghai Advanced Research Institute; TFCS: Test facility cryogenic system; ACCS: Accelerator cryogenic system; UNCS: Undulator cryogenic system; EPIC S: Experimental Physics and Industrial Control System; ACCP: Accelerator cryoplants; PV: Process Variables; CA: Channel Access; GUI: Graphical User Interfaces; IE: Industrial Ethernet; DCS: Distribution Control System; VTC: Vertical test cryostats; HTB: Horizontal test benches; CM: Cryogenic modules; UTC: Undulator test cryostat; BTC: Backup test cryostat; 
CPLD: Complex Programmable Logic Device; SNL: State Notation Language; CSS: Control System Studio; RAID: Redundant Arrays of Independent Disks

\section{Acknowledgements}

No

Authors' contributions

Geyang Jiang conceived of the presented idea and wrote the manuscript. Qing Ni, Shuhua Wang and Jiuce Sun contributed to the design and implementation. All authors discussed the results and contributed to the final manuscript. Zhengrong Ouyang supervised the project. The author(s) read and approved the final manuscript.

Funding

Project supported by Shanghai Municipal Science and Technology Major Project.

Availability of data and materials

Not applicable.

\section{Declarations}

Competing interests

No competing interets.

\section{Author details}

'Shanghai Advanced Research Institute, Chinese Academy of Sciences, No. 99 Haike Road, Zhangjiang Hi-Tech Park, Pudong, Shanghai, China. ${ }^{2}$ ShanghaiTech University, No. 393 Middle Huaxia Road, Pudong, Shanghai, China.

Received: 7 February 2021 Accepted: 13 May 2021

Published online: 23 July 2021

\section{References}

1. SHINE. Cryogenic system conceptual design report, ShanghaiTech University. Report number: Revision20190618. 2019.

2. Siemens AG. Industry network switches SCALANCE X manual. Siemens AG. Report number: 12/2019 C79000-G8976C187-32; 2019.

3. Lattice Semiconductor Corp. MachXO Family Data Sheet. Lattice Semiconductor Corp. Report number: DS1002 Version 03.0. 2013.

\section{Publisher's Note}

Springer Nature remains neutral with regard to jurisdictional claims in published maps and institutional affiliations.

\section{Submit your manuscript to a SpringerOpen ${ }^{\circ}$ journal and benefit from:}

- Convenient online submission

- Rigorous peer review

- Open access: articles freely available online

High visibility within the field

- Retaining the copyright to your article

Submit your next manuscript at $>$ springeropen.com 